\title{
Evaluación de la integración financiera entre los países pertenecientes al Mercado Integrado Latinoamericano
}

\author{
Duarte-Duarte, Juan Benjamín ; Garcés-Carreño, Laura Danielab; Vargas-Ayala, \\ Silvia Juliana ${ }^{c}$ y Vásquez-González, Valentina María ${ }^{d}$
}

\begin{abstract}
${ }^{a}$ Ingeniero Industrial. Doctor en Finanzas de Empresas. Profesor titular de la Universidad Industrial de Santander, Facultad de Ingenierías Físico Mecánicas, Escuela de Estudios Industriales y Empresariales, Bucaramanga, Colombia. Dirección de correspondencia: carrera 27 con calle 9, Ciudad Universitaria, Edificio de Ingeniera Industrial, oficina 207-25, Bucaramanga, Colombia. Correo electrónico: jduarte@,uis.edu.co; jbduarted@hotmail.com

${ }^{\mathrm{b}}$ Ingeniera Industrial. Magister en Ingeniería Industrial de la Universidad Industrial de Santander, Bucaramanga, Colombia. Profesora auxiliar de la Universidad Industrial de Santander. Docente investigadora de la Universidad Manuela Beltrán. Correo electrónico: lauradgarces@gmail.com

${ }^{c}$ Candidata a Ingeniera Industrial de la Universidad Industrial de Santander, Bucaramanga, Colombia. Correo electrónico: silviajayala@gmail.com

d Candidata a Ingeniera Industrial de la Universidad Industrial de Santander, Bucaramanga, Colombia. Correo electrónico: valevas97@hotmail.com
\end{abstract}

\begin{abstract}
The Mercado Integrado Latinoamericano - MILA - was created to promote the development of the countries that form it, by using a single mediator to carry out dealings across the different stock markets, thus removing transactional barriers that promote greater capital flow between the economies of Chile, Colombia, México and Peru, with the purpose to increase their competitiveness. The present work focusses on assessing the financial integration between the MILA's countries in the period between April 2008 and March 2018, based on the data from the most representative stock exchange of each country and using an improved correlations methodology by including statistical inference, which despite of providing better tools for the analysis doesn't result entirely conclusive mainly because the level of the mean correlations is lower than $50 \%$.
\end{abstract}

Keywords: Financial integration, Mercado Integrado Latinoamericano, stock market, correlations. 


\section{Resumen}

El Mercado Integrado Latinoamericano - MILA - fue creado con el fin de fomentar el desarrollo de los mercados de renta variable de los paises miembros, a través de un único mediador para la realización de transacciones entre los diferentes mercados de valores, eliminando barreras transaccionales, que promuevan un mayor flujo de capitales entre las economías de Chile, Colombia, México y Perú, con el fin de aumentar su competitividad. El presente trabajo se enfoca en evaluar la integración financiera entre los países que pertenecen al MILA en el periodo comprendido entre abril de 2008 y marzo de 2018 con base en los datos de las bolsas de valores más representativas de cada país y haciendo uso de una metodología de análisis de correlaciones mejorada a partir inferencia estadística, la cual a pesar de brindar mejores herramientas a la hora de realizar el análisis, no resulta del todo concluyente, debido a que los valores de las correlaciones promedio no alcanzan ni siquiera el $50 \%$.

Palabras clave: Integración financiera, Mercado Integrado Latinoamericano, mercado bursátil, correlaciones.

\section{Introducción}

En la actualidad, algunas regiones buscan seguir el camino de la unión europea integrando su economía a otras similares, con el propósito de aprovechar las ventajas asociadas a este tipo de integración como lo son el incremento de la eficiencia interna, una mayor solidez ante posibles shocks ocasionados por acontecimientos externos y a su vez alcanzar una convergencia monetaria, para lo cual es importante la existencia de integración financiera entre los países involucrados según da Silva Bichara (2001). En cuanto a esta, es de gran importancia evaluarla para los mercados bursátiles, es decir, las bolsas de valores, teniendo en cuenta que los mercados de renta variable son los que facilitan a los inversionistas la realización de transacciones generando impactos positivos en las finanzas de un país.

Considerando lo expuesto anteriormente, el propósito del presente trabajo es realizar una evaluación preliminar de la integración financiera entre los países que conforman el MILA (Chile, Colombia, México y Perú). Para ello se hace uso de una metodología de análisis de correlaciones en la que se propone una mejora que incorpora la inferencia estadística de tal manera que se obtengan resultados menos intuitivos y más robustos, a partir de las 
rentabilidades diarias de los principales índices de cada uno de los mercados objeto de estudio, durante el periodo comprendido entre abril de 2008 y marzo de 2018.

El siguiente trabajo está estructurado iniciando con la introducción, seguido de cinco secciones: en la primera se hace una breve revisión de la literatura basada en artículos o documentos resultantes de investigaciones previas, en la sección dos se exponen los datos utilizados, en la sección tres se explica la metodología, en la cuatro se analizan los resultados y por último se presentan las conclusiones.

\section{Revisión de la literatura}

El Mercado Integrado Latinoamericano nace como un acuerdo entre las bolsas de valores de los países pertenecientes a la Alianza del Pacifico (MILA, s.f.), con el fin de apoyar el proceso de integración de los mercados de renta variable y por tanto de las economías de estos países, brindando una mayor estabilidad que permita alcanzar mayores niveles de competitividad (Bolsa de Valores de Lima, 2018), lo cual eventualmente tiene como propósito la conversión de las economías en un área monetaria óptima, que permita la unificación monetaria, que implica el cumplimiento de unos criterios adicionales al de integración financiera, tal como se muestra en la Figura 1.

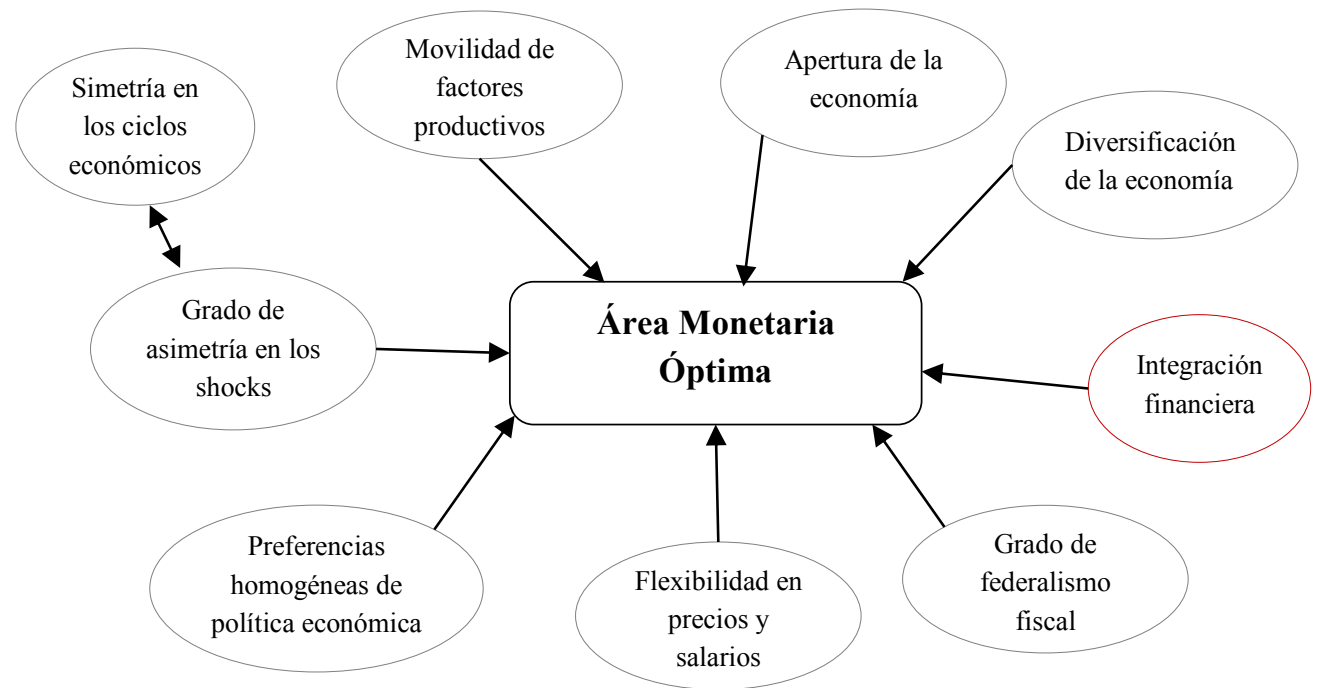

Figura 1. Criterios para la existencia de un área monetaria óptima. Fuente: Elaboración propia con información tomada de Zschiesche (2003). 
Evaluación de la integración financiera entre los paises pertenecientes al Mercado Integrado Latinoaméricano

Con relación al tema de integración financiera, se han realizado diferentes investigaciones a lo largo del tiempo, para diferentes economías del mundo, en las cuales se han obtenido importantes hallazgos. En la Tabla 1 se relacionan las principales metodologías junto con algunos de sus exponentes.

Tabla 1. Metodologías para la evaluación de integración financiera

\begin{tabular}{cc}
\hline Metodología & Autores \\
\hline & Chevallier, Nguyen, Siverskog y Uddin (2018) \\
Modelos multivariados & Yu Kabir (2008), Voa y Ellis (2018) \\
Rizvi y Arshad (2017) \\
Majdoub \& Mansour (2014) \\
Análisis de co-integración & Bundoo (2017), Click \& Plummer (2015) \\
& Majdoub, Mansour y Jouini (2016) \\
& Syllignakis y Kouretas (2010), Patel (2017) \\
& Al Nasser y Hajilee (2016), Bentes (2015) \\
Mínima distancia de los SDF & Ayuso y Blanco (1999), Chen y Knez (1995) \\
& Yao, He, Chen y Ou (2018) \\
& Longin y Solnik (1995), Le (1991), Glaser (2012) \\
Correlaciones & Min, Yank y Kolari (2003), Patel (2017) \\
& Meric, Nygren, Bentley y McCall (2015) \\
& Ayuso y Blanco (1999), Click \& Plummer (2005) \\
\hline
\end{tabular}

Fuente: Elaboración propia.

El presente documento es el resultado de un estudio premilinar, basado en la metodología de análisis de correlaciones, de un trabajo de investigación sobre integración financiera, en el cual posteriormente se aplicaran otras de las metodologías mencionadas en la Tabla 1.

Haciendo énfasis en la metodología de análisis de correlaciones planteada para el estudio preliminar, Longin y Solnik (1995) afirman que la creciente integración internacional conduce a un incremento progresivo de la correlación entre mercados. Además, sugieren que los períodos de alta volatilidad suelen generar mayor grado de correlación, lo cual es respaldado por Min,Yank y Kolari (2003), quienes aseguran que el grado de integración financiera entre diferentes países tiende a variar con el tiempo, especialmente en aquellos períodos que enmarcan crisis financieras; afirmando, a partir de esto, que en general los mercados resultan estar más integrados después de una crisis que antes de presentarse la misma, tal como encontraron Meric, Nygren, Bentley y McCall (2015) en su investigación, basados en los resultados de los estudios empíricos realizados por Le (1991) en los que se muestra que la correlación entre mercados bursátiles europeos aumentó y por su parte, la diversificación de la cartera global disminuyó significativamente después de la quiebra del mercado bursátil mundial de 1987, en el cual comparan series de correlaciones entre el mercado de acciones estadounidense y el de 20 países europeos encontrando niveles de correlación más altos para el período de poscrisis en todos los casos. A partir de esto, afirman que los movimientos conjuntos de los mercados bursátiles de Estados Unidos y 
Europa están sustancialmente más cercanos después de la caída bursátil del 2008 que antes del declive, basados también en la premisa de que altos niveles de correlaciones promedio indican que un mercado de acciones se encuentra muy bien integrado con otro. Por otra parte, Glaser (2012) concluye que los coeficientes de correlación entre diferentes economías de todo el mundo, especialmente economías emergentes, al ser analizados, para un solo periodo que involucra los últimos 15 años, muestran valores bajos, los cuales al reducirse el rango del periodo de análisis reflejan incrementos que sugieren que estos mercados se han ido desarrollando de manera más cercana en los últimos tiempos, gracias a factores tales como la globalización, la apertura de nuevas economías, los incentivos a la inversión extranjera y las crisis financieras. Finalmente, Ayuso y Blanco (1999) en su investigación, en la cual calcularon las correlaciones para New York, Londres, Paris, Madrid, Frankfurt, Milán y Tokio en los periodos comprendidos entre 1990-1994 y 1995 1999, encontraron que en el segundo periodo las correlaciones son mayores, lo cual puede sugerir una mayor integración entre estos mercados. Sin embargo, al igual que Longin y Solnik (1995), aclaran que este criterio no es suficiente debido a que es muy intuitivo, pues incluso cuando dos mercados están totalmente integrados, es decir, no existen oportunidades de arbitraje, los retornos de un activo cuentan con dos componentes, uno común para todos y uno que depende de la idiosincrasia y este puede llegar a ser tan fuerte como para hacer que las correlaciones sean bajas.

\section{Datos}

Los datos utilizados para el desarrollo de la investigación fueron los precios diarios, para el periodo comprendido entre abril del 2008 y marzo del 2018, de los índices generales (IPSA, COLCAP, IPC e IGBVL/Perú General) para cada uno de los diferentes mercados que conforman el MILA. Estos datos se obtuvieron de bases de datos representativas de cada país, las cuales fueron:

Chile: Base de datos Economatica

Colombia: Bolsa de valores de Colombia

México: S\&P Dow Jones Índices México

Perú: Bolsa de valores de Lima (2008-2014) e Investing (2015-2018)

Con el propósito de hacer que los precios y las rentabilidades sean comparables entre países, se tomaron como base las fechas de cotización del mercado mexicano, asumiendo como precio de cierre el precio de la última fecha de cotización para los días en los que alguno de los mercados se encontraba cerrado. 


\section{Metodología}

Para determinar si existe integración financiera entre los cuatro países objeto de estudio, así como los cambios generados en esta debido a la creación del MILA y posteriormente la inclusión de México dentro del mismo, en el presente trabajo se hace uso de una metodología de análisis de correlaciones, que a diferencia de la utilizada hasta el momento por otros autores, introduce una mejora en el análisis partiendo de la inferencia estadística que involucra una clasificación por terciles, lo que complementa el análisis de correlaciones intuitivo utilizado por autores previos buscando hacerlo más robusto. Para esto, es necesario estimar la rentabilidad continua diaria de los precios para los principales indices de cada una de las bolsas de valores estudiadas (ver ecuación 1).

$$
R_{t}=\ln \left(\frac{P_{t}}{P_{t-1}}\right)
$$

Donde, $\mathrm{R}_{\mathrm{t}}$ es la rentabilidad continua en el día $t, \mathrm{P}_{\mathrm{t}}$ es el precio en el día de cotización $\mathrm{t}$ y $\mathrm{P}_{\mathrm{t}-1}$ es el precio en el día de cotización $\mathrm{t}-1$.

Con las rentabilidades halladas, se calculan y grafican las correlaciones trimestrales para los siguientes periodos de tiempo: Periodo total [Abril de 2008 - Marzo de 2018], antes de la creación del MILA [Abril de 2008 - Marzo de 2011], durante la operación del MILA sin incluir a México [Abril de 2011 - Marzo de 2014], y después de la inclusión de México en este [Abril de 2014 - Marzo de 2018].

Luego, en el periodo total para evaluar la igualdad tanto entre rentabilidades como entre correlaciones, se realizan dos pruebas haciendo uso del software estadístico Minitab.

Para las rentabilidades, en primera instancia se realiza una prueba de normalidad verificando en@Risk las distribuciones que más se ajustan a los datos, obteniendo los resultados que se muestras en la Tabla 2 .

Tabla 2. Distribuciones que presentan mayor ajuste

\begin{tabular}{cccccccc}
\hline \multicolumn{2}{c}{ México } & \multicolumn{2}{c}{ Perú } & \multicolumn{2}{c}{ Colombia } & \multicolumn{2}{c}{ Chile } \\
\hline Distribución & $\begin{array}{c}\text { Chi- } \\
\text { cuadrado }\end{array}$ & Distribución & $\begin{array}{c}\text { Chi- } \\
\text { cuadrado }\end{array}$ & Distribución & $\begin{array}{c}\text { Chi- } \\
\text { cuadrado }\end{array}$ & Distribución & $\begin{array}{c}\text { Chi- } \\
\text { cuadrado }\end{array}$ \\
Logistic & 112.38 & Logistic & 265.37 & Logistic & 340.30 & Logistic & 178.95 \\
Loglogistic & 113.05 & Normal & 705.79 & Normal & 618.73 & Loglogistic & 178.95 \\
\hline InvGauss & 352.59 & & & & & Normal & 448.28 \\
Normal & 353.94 & & & & & & \\
\hline
\end{tabular}

Fuente: Elaboración propia. El criterio de ajuste de chi-cuadrado establece que entre menor sea su valor, mejor es el ajuste de la distribución.

Puesto que la distribución normal es una de las que mejor se ajusta para todos los casos posicionándose entre los primeros cuatro lugares, se puede asumir normalidad para los 
datos y aplicar la prueba $\mathrm{t}$ de dos muestras con el fin de verificar igualdad estadística entre las medias de las diferentes series de datos. Para esto, la prueba plantea como hipótesis nula y alterna las expresiones que se muestran a continuación:

$$
\begin{gathered}
H_{0}: \mu_{l}-\mu_{2}=0 \\
H_{1}: \mu_{l}-\mu_{2} \neq 0
\end{gathered}
$$

Donde $\mu$ es la media de la población. Además, hace uso del estadístico de prueba t para aplicar la regla de decisión, con un nivel de confianza del $95 \%$, que permite rechazar o no la hipótesis nula planteada.

En el caso de las correlaciones, considerando que el tamaño de la muestra es pequeño, de tan solo 40 datos para cada serie, no es posible asumir normalidad y por lo tanto se aplica una prueba no paramétrica que permita verificar igualdad estadística entre las medianas de las distintas correlaciones, tal como es la prueba de Mann Whitney. Para esto, la prueba plantea como hipótesis nula y alterna las siguientes expresiones:

$$
\begin{aligned}
& H_{0}: \eta_{1}=\eta_{2} \\
& H_{1}: \eta_{1} \neq \eta_{2}
\end{aligned}
$$

Donde $\eta$ es la mediana de la población. Además, hace uso del estadístico de prueba U para aplicar la regla de decisión, con un nivel de confianza del 95\%, que permite rechazar o no la hipótesis nula planteada.

Los p valores obtenidos, para cada una de las pruebas de manera independiente, al igual que los promedios de las correlaciones son organizados en orden ascendente y se clasifican en terciles con el propósito de facilitar el análisis de los resultados.

Por otro lado, a partir de las gráficas de las correlaciones calculadas para los tres periodos de tiempo que involucran al MILA, se realiza un análisis de observación con el objetivo de identificar posibles cambios generados como consecuencia de la creación del MILA o de la posterior inclusión de México en este mercado.

\section{Resultados}

En la Tabla 3. se muestran los resultados, límites del intervalo de confianza, el estadístico de prueba y el $\mathrm{P}$ valor, de la prueba $\mathrm{t}$ de dos muestras aplicada a las rentabilidades de todas las posibles combinaciones entre los diferentes países, los cuales se encuentran clasificados en tres niveles, bajo, medio y alto, obtenidos mediante el cálculo de terciles con base en los $\mathrm{P}$ valores resultantes. 
Evaluación de la integración financiera entre los países pertenecientes al Mercado Integrado Latinoaméricano

Tabla 3. Resultados de la prueba t de dos muestras para las rentabilidades

\begin{tabular}{lcccc}
\hline Rentabilidades & LI IC & LS IC & T & P valor \\
\hline PERU GENERAL-COLCAP & -0.000886 & 0.000513 & -0.52 & 0.601 \\
PERU GENERAL-IPSA & -0.000833 & 0.000574 & -0.36 & 0.719 \\
\hline IPC-COLCAP & -0.000687 & 0.000515 & -0.28 & 0.779 \\
COLCAP-IPSA & -0.000642 & 0.000842 & 0.26 & 0.791 \\
\hline IPC-PERU GENERAL & -0.000610 & 0.000496 & -0.20 & 0.839 \\
IPC-IPSA & -0.000635 & 0.000577 & -0.09 & 0.926 \\
\hline
\end{tabular}

Fuente: Elaboración propia. Los $\mathrm{p}$ valores obtenidos para cada una de las pruebas $\mathrm{t}$ realizadas están clasificados en terciles identificados cada uno con diferente color; el rojo, naranja y verde representan los niveles bajo, medio y alto respectivamente.

Basados en los resultados de la prueba t realizada para todas las combinaciones de países, se evidencia que las medias de las rentabilidades de todos los países miembros del MILA son estadísticamente iguales, además de existir una relación directa entre la igualdad de las medias y los niveles promedio de correlación presentados en la Tabla 4., en la cual se encuentran las estadísticas básicas para las diferentes combinaciones de países, además de la clasificación en tres niveles, bajo, medio y alto, obtenidos mediante el cálculo de terciles.

Tabla 4. Terciles y estadísticas básicas para las correlaciones

\begin{tabular}{lcccc}
\hline Correlaciones trimestrales & Media & Desviación & Min & Max \\
\hline PERU GENERAL-COLCAP & 0.33102679 & 0.17809099 & -0.03198601 & 0.73831564 \\
PERU GENERAL-IPSA & 0.37758456 & 0.20438544 & -0.13671043 & 0.76227261 \\
\hline IPC-COLCAP & 0.37914082 & 0.17522890 & -0.02998492 & 0.68133920 \\
COLCAP-IPSA & 0.39155326 & 0.17119331 & 0.05183905 & 0.72632736 \\
\hline IPC-PERU GENERAL & 0.39961620 & 0.19571358 & -0.06745895 & 0.72632085 \\
IPC-IPSA & 0.48768867 & 0.16345975 & 0.05857511 & 0.76932500 \\
\hline
\end{tabular}

Fuente: Elaboración propia. Las medias halladas para las correlaciones se encuentran clasificadas en terciles identificados cada uno con diferente color; el rojo representa el nivel bajo, el naranja el nivel medio y el verde el nivel alto.

En la Tabla 4 se observa que en general las medias de las correlaciones trimestrales son cercanas entre sí para todos los casos, encontrándose en un rango entre 0.33 y 0.49 el cual aunque se ve afectado por relaciones de tipo inversa en algunas de las combinaciones, es contrarrestado por una magnitud alta y similar en cuanto a relaciones directas. Además, se evidencia que las correlaciones más bajas se presentan entre Perú con Colombia y Chile respectivamente mientras que las correlaciones más altas se encuentran entre México con Chile y Perú correspondientemente.

En la Tabla 5. se presentan los resultados, límites del intervalo de confianza, el estadístico de prueba y el P valor, de la prueba de Mann Whitney aplicada a pares de correlaciones de las distintas combinaciones entre los países objeto de estudio. Estos resultados se 
encuentran clasificados en tres niveles, bajo, medio y alto, obtenidos mediante el cálculo de terciles con base en los $\mathrm{P}$ valores resultantes.

Tabla 5. Resultados de la prueba de Mann-Whitney para cada par de correlaciones

\begin{tabular}{lcccc}
\hline Correlaciones & LI IC & LS IC & W & P valor \\
\hline IPC-IPSA; PERU GEN-COLCAP & 0.075256 & 0.236952 & 2001 & 0.000 \\
IPC-COLCAP; IPC-IPSA & -0.183180 & -0.024483 & 1340 & 0.007 \\
IPC-IPSA; PERU GEN-IPSA & 0.018114 & 0.190093 & 1868 & 0.017 \\
IPC-IPSA; COLCAP-IPSA & 0.011852 & 0.175084 & 1863 & 0.020 \\
IPC-PERU GEN; IPC-IPSA & -0.172398 & 0.000733 & 1418 & 0.053 \\
\hline PERU GEN-COLCAP; COLCAP-IPSA & -0.147346 & 0.017958 & 1461 & 0.127 \\
IPC-PERU GEN; PERU GEN-COLCAP & -0.018092 & 0.159654 & 1778 & 0.130 \\
PERU GEN-COLCAP; PERU GENL-IPSA & -0.143786 & 0.035800 & 1488 & 0.206 \\
IPC-COLCAP; PERU GEN-COLCAP & -0.030058 & 0.142158 & 1751 & 0.209 \\
IPC-PERU GEN; PERU GEN-IPSA & -0.073164 & 0.112182 & 1658 & 0.718 \\
\hline IPC-PERU GEN; IPC-COLCAP & -0.064791 & 0.110602 & 1654 & 0.747 \\
IPC-COLCAP; COLCAP-IPSA & -0.095448 & 0.067959 & 1594 & 0.806 \\
PERU GEN-IPSA; COLCAP-IPSA & -0.096555 & 0.074424 & 1595 & 0.814 \\
IPC-PERU GEN; COLCAP-IPSA & -0.080494 & 0.095024 & 1642 & 0.836 \\
IPC-COLCAP; PERU GEN-IPSA & -0.092445 & 0.086865 & 1611 & 0.935 \\
\hline
\end{tabular}

Fuente: Elaboración propia. Los $\mathrm{p}$ valores obtenidos para cada una de las pruebas de Mann Whitney realizadas están clasificados en terciles identificados cada uno con diferente color; el rojo, naranja y verde representan los niveles bajo, medio y alto respectivamente.

Con base en los resultados de la prueba de Mann Whitney realizada para todas las posibles combinaciones de correlaciones, se puede determinar que la mediana de las correlaciones entre IPC e IPSA es significativamente diferente a la mediana de cada uno de los demás pares de correlaciones, excepto la de IPC con Perú General con la cual a pesar de no presentar diferencia significativa, no son tan cercanas entre sí debido a que la diferencia entre el $\mathrm{p}$ valor obtenido y el nivel de significancia (5\%) es minima $(0.3 \%)$.

Por otro lado, se encuentra que las correlaciones entre los pares IPC-COLCAP y PERU GENERAL-IPSA son las que cuentan con una menor diferencia estadística en sus medianas, lo cual podría sugerir existencia de integración financiera entre los cuatro mercados involucrados. En la Figura 2 y la Figura 2. Correlaciones IPC-IPSA vs PERÚ-COLCAP Figura 3. Correlaciones IPC-COLCAP vs PERÚ-IPSAse observa que las correlaciones con medianas estadísticamente iguales se encuentran más cercanas entre si y presentan comportamientos similares en una mayor cantidad de periodos en comparación de aquellas en las que no se presenta igualdad de medianas. Sin embargo, a diferencia de lo que se puede inferir del $\mathrm{p}$ valor de 93,5\% obtenido de la prueba Mann Whitney, al observar la grafica, las curvas no presentan exactamente la misma tendencia, restando validez al supuesto de integración entre los cuatro países miembros del MILA, ya que sus comportamientos no parecen reflejar dependencia o causalidad directa entre si. 
Evaluación de la integración financiera entre los países pertenecientes al Mercado Integrado Latinoaméricano

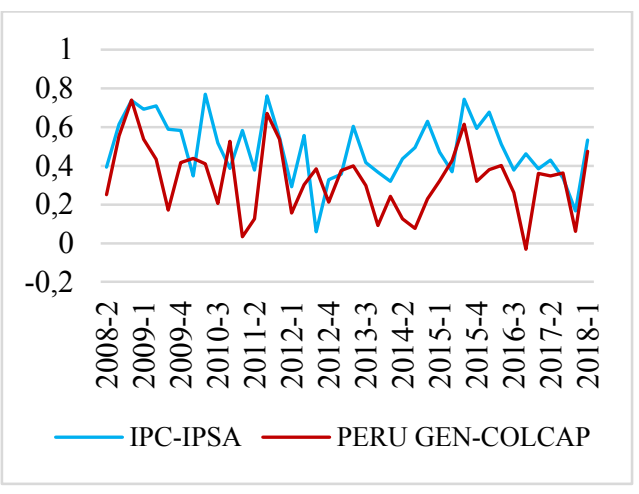

Figura 2. Correlaciones IPC-IPSA vs PERÚ-COLCAP Fuente: Elaboración propia

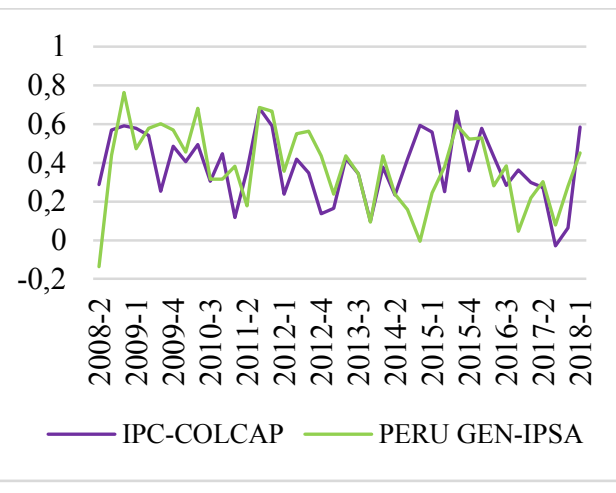

Figura 3. Correlaciones IPC-COLCAP vs PERÚ-IPSA Fuente: Elaboración propia

De acuerdo con la información que se presenta en la Tabla 6. se encuentra que con la creación del MILA se generaron cambios positivos para la mayoría de los pares de correlaciones, indicando esto una mayor integración entre los cuatro países incluyendo a México, aun cuando este no era inicialmente miembro. Sin embargo, después de la inclusión de México en el Mercado Integrado Latinamericano se evidencia un alejamiento en las medianas de las correlaciones siendo los cambios negativos los más representativos para este periodo.

Tabla 6. Resultados de la prueba de Mann-Whitney para los tres periodos de tiempo estudiados

\begin{tabular}{llcc}
\hline \multicolumn{1}{c}{ Correlaciones } & $\begin{array}{c}\text { ANTES } \\
\text { P valor }\end{array}$ & $\begin{array}{c}\text { SIN MX } \\
\text { P valor }\end{array}$ & $\begin{array}{c}\text { CON MX } \\
\text { P valor }\end{array}$ \\
\hline IPC-PERU GEN; IPC-COLCAP & 0,0606 & $\uparrow 0,7950$ & $\downarrow 0,2503$ \\
IPC-PERU GEN; IPC-IPSA & 0,7508 & $\downarrow 0,5444$ & $\downarrow 0,0136$ \\
IPC-PERU GEN; PERU GEN-COLCAP & 0,0531 & $\uparrow 0,5067$ & $\uparrow 0,8065$ \\
IPC-PERU GEN; PERU GEN-IPSA & 0,2366 & $\uparrow 0,6236$ & $\uparrow 0,8653$ \\
IPC-PERU GEN; COLCAP-IPSA & 0,0166 & $\uparrow 0,7508$ & $\downarrow 0,0332$ \\
\hline IPC-COLCAP; IPC-IPSA & 0,0194 & $\uparrow 0,5067$ & $\downarrow 0,0864$ \\
IPC-COLCAP; PERU GEN-COLCAP & 0,5067 & $\uparrow 0,5834$ & $\downarrow 0,3558$ \\
IPC-COLCAP; PERU GEN-IPSA & 0,4705 & $\downarrow 0,3408$ & $\downarrow 0,2503$ \\
IPC-COLCAP; COLCAP-IPSA & 0,4705 & $\uparrow 0,9770$ & $\downarrow 0,3964$ \\
IPC-IPSA; PERU GEN-COLCAP & 0,0351 & $\uparrow 0,1749$ & $\downarrow 0,0021$ \\
IPC-IPSA; PERU GEN-IPSA & 0,1124 & $\uparrow 0,8399$ & $\downarrow 0,0079$ \\
IPC-IPSA; COLCAP-IPSA & 0,0086 & $\uparrow 0,4705$ & $\uparrow 0,5095$ \\
PERU GEN-COLCAP; PERU GEN-IPSA & 0,2855 & $\downarrow 0,1572$ & $\uparrow 0,8653$ \\
PERU GEN-COLCAP; COLCAP-IPSA & 0,9770 & $\downarrow 0,7075$ & $\downarrow 0,0438$ \\
PERU GEN-IPSA; COLCAP-IPSA & 0,2602 & $\uparrow 0,4025$ & $\downarrow 0,0302$ \\
\hline
\end{tabular}

Fuente: Elaboración propia. En la tabla se encuentra los p valores obtenidos en cada prueba de Mann Whitney en los tres periodos definidos (antes del MILA-ANTES, durante la operación del MILA sin México-SIN MX y durante la operación del MILA con México-CON MX), los cuales son comparados con 
respecto al periodo anterior, identificando con flechas verdes hacia arriba los cambios positivos y con flechas rojas hacia abajo los negativos.

No obstante, en la Figura 4 se puede observar como a partir de la creación del MILA, se presenta en promedio una disminución en el valor de las correlaciones con respecto al periodo anterior, con lo cual a pesar de tener comportamientos similares no es posible asegurar la existencia de una mayor integración financiera entre los cuatro países. Por otra parte, para el tercer periodo analizado se encuentra una diferencia notable en el comportamiento de las correlaciones, alcanzando en algunos de los trimestres los valores más bajos de las correlaciones para todo el horizonte de tiempo contemplado.

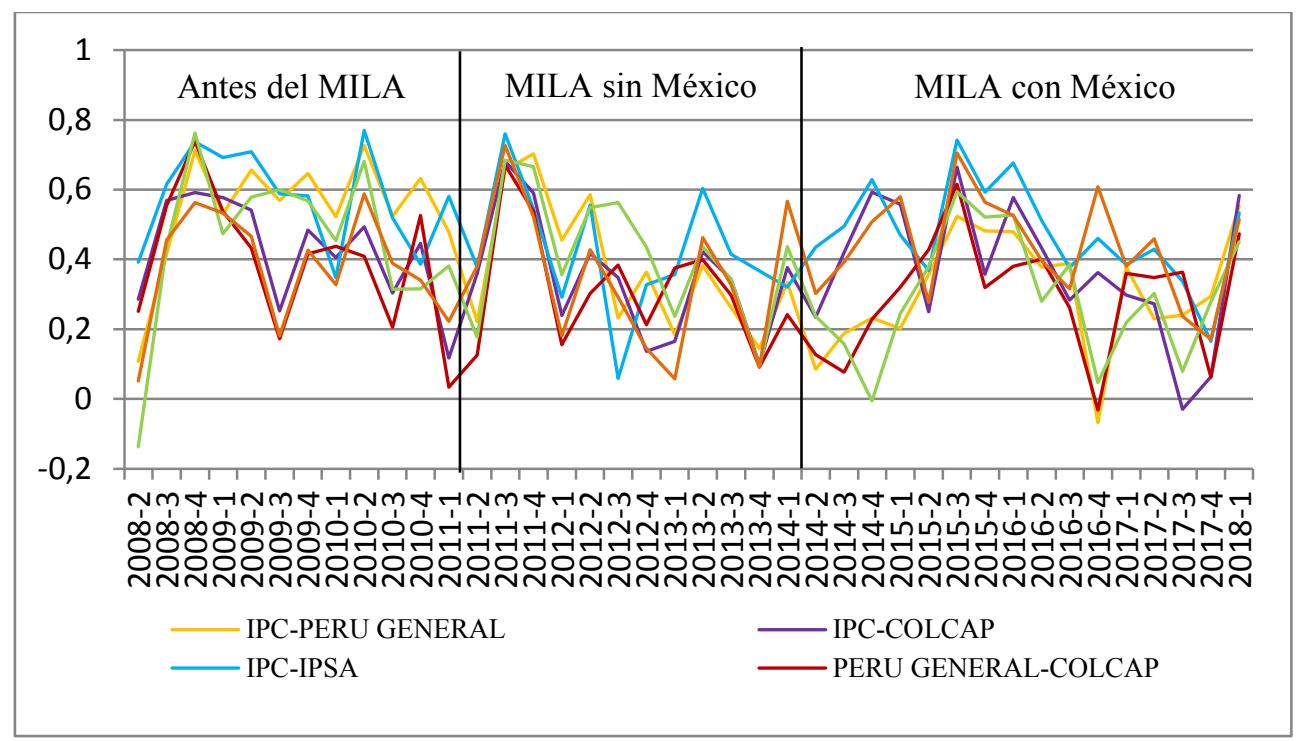

Figura 4. Correlaciones antes y después del MILA. Fuente: Elaboración propia.

\section{Conclusiones}

El MILA fue creado en el 2011 con el objetivo de integrar los mercados bursátiles de los países que lo componen; sin embargo, a partir de los resultados obtenidos se evidencia que no se ha presentado ningún cambio relevante que muestre signos de una mayor integración, por el contrario se observa una disminución de esta especialmente a partir de la inclusión de México en la plataforma.

Siendo la creación del Mercado Integrado Latinoamericano un suceso importante al igual que la inclusión de México en este, durante el periodo de tiempo contemplado es posible que se presentaran acontecimientos, desligados del MILA, que afectan al mercado bursatil 
haciendo que el comportamiento de los cuatro mercados estudiados se dirija en una dirección contraria a la de integración propuesta por el MILA.

De acuerdo con los resultados no se puede asegurar que entre Chile, Colombia, Perú y México se presenta una integración financiera; tampoco es posible afirmar que sí existe únicamente para ciertas parejas de países, teniendo en cuenta que las correlaciones promedio halladas para cada una de ellas no muestran valores cercanos al 100\%.

La metodología aplicada a pesar de contar con un alto nivel de inferencia estadística, lo cual brinda mejores herramientas a la hora de realizar el análisis, no resulta del todo concluyente, debido a que los valores de las correlaciones promedio no alcanzan ni siquiera el $50 \%$.

\section{Referencias}

Al Nasser, M., \& Hajilee, M. (2016). Integration of emerging stock markets with global stock markets. Research in International Business and Finance, 36, 1-12.

Ayuso, J., \& Blanco, R. (1999). Has financial market integration increased during the nineties? España: Banco de España.

Bentes, S. (2015). On the integration of financial markets: How strong is the evidence from five international stock markets? Physica A: Statistical Mechanics and its Applications, 429(1), 205214.

Bolsa de Valores de Lima. (Junio de 2018). Bolsa de Valores de Lima. Obtenido de http://www.bvl.com.pe/mila/preguntas_frecuentes.pdf

Bundoo, S. (2017). Stock market development and integration in SADC (Southern African Development Community). Review of Development Finance, 7, 64-72.

Chen, Z., \& Knez, P. (1995). Measurement of Market Integration and Arbitrage. Review of Financial Studies, 8(2), 287-325.

Chevallier, J., Nguyen, D., Siverskog, J., \& Uddin, G. (2018). Market integration and financial linkages among stock markets in Pacific Basin countries. Journal of Empirical Finance, 4, 77-92.

Click, R., \& Plummer, M. (2015). Stock market integration in ASEAN after the Asian financial crisis. Journal of Asian Economics, 16(1), 5-28.

da Silva Bichara, J. (2001). Coordinación de política macroeconómica en el Mercosur. Madrid: Universidad Autónoma de Madrid.

Glaser, J. (12 de Noviembre de 2012). Morningstar. Obtenido de http://www.morningstar.co.uk/uk/news/96521/why-have-global-correlations-increased.aspx

Le, S. (1991). International investment diversification before and after the October 19, 1987 stock market crisis. Journal of Business Research, 22(4), 305-310. 
Longin, F., \& Solnik, B. (Febrero de 1995). Is the correlation in international equity returns constant: 1960-1990? Journal of International Money and Finance, 14(1), 3-26.

Majdoub, J., \& Mansour, W. (2014). Islamic equity market integration and volatility spillover between emerging and US stock markets. North American Journal of Economics and Finance, $29,452-470$.

Majdoub, J., Mansour, W., \& Jouini, J. (2016). Market integration between conventional and Islamic stock prices. North American Journal of Economics and Finance, 37, 436-457.

Meric, I., Nygren, L., Bentley, J., \& McCall, C. (2005). Co-Movements Of U.S. And European Stock Markets Before And After The 2008 Gloal Stock Market Crash. Studies in Business and Economics, 10(2), 83-98.

MILA. (s.f.). MILA. Recuperado el 15 de Junio de 2018, de https://mercadomila.com/quienessomos/resena-historica/

Min, I., Yang, J., \& Kolari, J. (Febrero de 2003). Stock Market Integration and Financial Crises: The Case of Asia. Applied Financial Economics, 13(7), 477-486.

Patel, R. (2017). Co-Movement and Integration Among Stock Markets : A Study of 14 Countrie. Indian Journal of Finance, 11(9).

Rizvi, S., \& Arshad, S. (2017). Analysis of the efficiency-integration nexus of Japanese stock market. Physica A: Statistical Mechanics and its Applications, 470, 296-308.

Syllignakis, M., \& Kouretas, G. (2010). German, US and Central and Eastern European Stock Market Integration. Revisión de Economías Abiertas, 21(4), 607-628.

Voa, X., \& Ellis, C. (2018). International financial integration: Stock return linkages and volatility transmission between Vietnam and advanced countries. Emerging Markets Review.

Yao, S., He, H., Chen, S., \& Ou, J. (2018). Financial liberalization and cross-border market integration: Evidence from China's stock market. International Review of Economics \& Finance.

Yu, J., \& Kabir, M. (2008). Global and regional integration of the Middle East and North African (MENA) stock markets. The Quarterly Review of Economics and Finance, 48(3), 482-504.

Zschiesche, J. (2003). España y el proceso de integración europeo: prevención y ajuste ante perturbaciones asimétricas. Una aproximación relativa a través de la teoría de las áreas monetarias óptimas. Madrid: Universidad Complutense de Madrid. 